\title{
Patient perspectives of care in a regionalised trauma system: lessons from the Victorian State Trauma System
}

\section{Belinda J Gabbe MAppSc, MBiostat, PhD, Associate Professo $\left(\right.$ Research) ${ }^{1}$ \\ Jude S Sleney $\mathrm{BSc}$ (Hons), MSc, Research Fellow ${ }^{2}$ \\ Cameron M Gosling $\operatorname{BAppSc}(\mathrm{HM})$ GradDip(ExRehab) MAppSc \\ Research Fellow \\ Krystle Wilson BN(Hons) Research Assistant \\ Melissa J Hart RN, Orth Cert, BEd Project Coordinator Victorian Orthopaedic Trauma Outcomes Registry \\ Ann M Sutherland \\ RN, AdvCertM'ment, GradDipOHE\& \\ Trauma Outcomes Coordinator \\ Nicola Christie $B A, P h D$ Senior Lecturer \\ 1 Department of Epidemiology an Preventive Medicine Monash University, \\ Melbourne, VIC \\ 2 Department of Sociology, University of Surrey, Guildford Surrey, UK. \\ 3 Centre for Transport Studies, Department of Civil, Environmental and Geomatic Engineering Unversity College London London, UK \\ belinda.gabbe@ monash.edu}

MJA 2013; 198: 149-152 doi: 10.5694/mjal2.11179

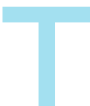
rauma is considered a "timesensitive disease", requiring integrated and timely treatment to reduce mortality and morbidity. ${ }^{1}$ There is strong evidence that organised trauma systems reduce the risk of mortality, ${ }^{2,3}$ and there is increasing evidence to suggest that trauma centre care is cost-effective, and improves patient function and quality of life. $^{4-6}$

After a review of trauma services in the state, $^{7}$ Victoria introduced an organised trauma system in $2000 .^{8}$ Over the past decade, there has been a reduction of risk-adjusted mortality ${ }^{2}$ and improvement in functional outcomes for major trauma patients in the state. ${ }^{4}$ However, disability is prevalent for trauma patients, even 12 months after injury. ${ }^{4,9}$ Trauma care delivery can be improved by understanding patients' experiences. ${ }^{10}$ The aim of this study was to investigate injured patients' experiences of trauma care to inform improvements in service delivery.

\section{Methods}

\section{Setting}

The Victorian State Trauma System (VSTS) coordinates prehospital and acute care across the state. All 138 trauma-receiving hospitals have a system level of designation. One paediatric and two adult hospitals are defined as major trauma services (MTS). The population-based Victorian State Trauma Registry (VSTR) collects data about all major trauma patients in Victoria. ${ }^{8}$ The Victorian Orthopaedic Trauma Outcomes Registry (VOTOR) is a sentinel site registry that collects data about adult orthopaedic trauma patients with a length of stay over 24 hours who are admitted to four hospitals. ${ }^{11}$ These registries enable monitoring of the VSTS. Survivors to discharge are followed up at 6, 12 (VSTR and VOTOR) and 24 months (VSTR) by telephone interview. ${ }^{12}$

\begin{abstract}
Objectives: To explore injured patients' experiences of trauma care to identify areas for improvement in service delivery.

Design, setting and participants: Qualitative study using in-depth, semistructured interviews, conducted from 1 April 2011 to 31 January 2012, with 120 trauma patients registered by the Victorian State Trauma Registry and the Victorian Orthopaedic Trauma Outcomes Registry and managed at the major adult trauma services (MTS) in Victoria.
\end{abstract}

Main outcome measures: Emergent themes from patients' experiences of acute, rehabilitation and post-discharge care in the Victorian State Trauma System (VSTS).

Results: Patients perceived their acute hospital care as high quality, although issues with communication and surgical management delays were common. Discharge from hospital was perceived as stressful, and many felt ill prepared for discharge. A consistent emerging theme was the sense of a lack of coordination of post-discharge care, and the absence of a consistent point of contact for ongoing management. Most patients' primary point of contact after discharge was outpatient clinics at the MTS, which were widely criticised because of substantial delays in receiving an appointment, prolonged waiting times, limited time with clinicians, lack of continuity of care and inability to see senior clinicians.

Conclusions: This study highlights perceived issues in the patient care pathway in the VSTS, especially those relating to communication, information provision and post-discharge care. Trauma patients perceived the need for a single point of contact for coordination of post-discharge care.

\section{Sampling strategy}

The following VSTR and VOTOR patients were eligible to participate:

- blunt trauma patients (over $90 \%$ of VSTR and VOTOR cases);

- patients at least 12 to 24 months after injury;

- patients aged 18 years or more;

- Transport Accident Commission (TAC) compensatable or non-compensatable patients; and

- patients who received definitive care at an adult MTS.

Purposive sampling was used to ensure the sample reflected the diversity of registry patients. ${ }^{13}$ The target was 120 participants with 60 of each sex, with even representation of registries, hospitals and compensatation status across three age groups (18-44, 45-64 and $\geqslant 65$ years). A balance of TAC and non-compensatable participants were sampled, as TAC patients are known to have poorer outcomes. ${ }^{4,9}$

The project was approved by the Alfred Health and Melbourne Health Human Research Ethics Committees.

\section{Data collection}

Demographic, injury and outcomes data were extracted from the VSTR and VOTOR. Individual in-depth, semi-structured telephone interviews were used to enable participants to speak freely about their experiences. ${ }^{14}$ Patients were invited to participate at the 12- or 24-month VSTR or VOTOR interview, and all the interviews were conducted between 1 April 2011 and 31 January 2012. Three experienced interviewers completed the interviews. ${ }^{14} \mathrm{~A}$ topic guide provided the interviewer with prompts about key issues for exploration. Topics relevant to this article are shown in Box 1 . Verbal consent was obtained and each interview was recorded using a digital voice recorder.

\section{Data analysis}

Interviews were transcribed from the audio recordings. Thematic analysis was used to identify important thematic groupings and the relationships between them. ${ }^{14}$ This involved (i) reading each transcript and listening 
1 Interviewer prompts used in semi-structured interviews with 120 trauma patients treated at major trauma services in Victoria

Injury treatment

- How do you feel about the care you received?

- How could the treatment you received be improved?

- What information or advice did you receive about your injury?

- If you did not receive information, what information would you have liked to have received and from whom?

Perception of recovery

- What helped the most?

- And the reverse, what if anything made it harder to recover?

- What would you like to have happened differently with your treatment?

to the recorded interview, if appropriate, to make sense of the interview data; and (ii) re-examining the transcript as a component of all interviews to make sense of what was being said by the participants as a group.

Twenty-six interviews were doublecoded to enable crosschecking of coding and interpretation of the data. ${ }^{13}$ Each researcher read the transcripts and generated and collated these codes into tentative themes. Four researchers discussed and developed the emerging coding frame before coding was completed. The coding frame was based on common topics, patterns and relationships emerging from the transcripts. Two researchers coded the remaining interviews, and one applied the frame to the coded transcripts, allowing for the themes in the coding frame to be revised and refined during the process of coding. The constant comparison method was used to ensure reliability. ${ }^{15}$ Transcripts were revisited a number of times to ensure consistency of meaning of individual responses.

The thematic analysis is exemplified by participants' quotes drawn from the transcripts.

\section{Results}

\section{Profile of participants}

There was undersampling in the older age group, a small gender imbalance and minor overrepresentation of VSTR patients and TAC clients in the study cohort (Box 2). Most participants reported no pre-injury disability, and $71 \%$ (85/120) were working or studying before their injury. More than half reported a "good recovery" on the Extended Glasgow Outcome Scale (GOS-E) (Box 3). Isolated lower extremity fractures were prevalent $(23 / 60)$ in
VOTOR participants. Twenty-seven of the 60 VSTR participants had sustained a traumatic brain injury (TBI), and of those, 20 were multitrauma patients.

\section{Hospital care}

Quality of care: Most participants reported positive or mixed experiences with their hospital care. Few participants reported only negative experiences. For major trauma patients, it was difficult to comment on the quality of hospital care because of their prolonged intensive care unit admissions, or because of post-traumatic amnesia after TBI. The overall impression of the hospital care received was positive, with a sense of being fortunate to receive high-quality care.

I've got nothing but praise. I was so well looked after and everyone was so supportive. ... I can't believe how well we are looked after in our country. (56-year-old woman, TAC, multiple injuries)

Delays: Treatment delays were common, and many perceived that these delays prolonged their time spent in hospital and their overall recovery. Delays to surgery were prevalent for orthopaedic trauma patients. These patients perceived their lesser injury severity to be a key factor in the delays.

it was ... days before I was operated, ...I had to fast the entire time because they kept saying, "Yeah, we'll do it today", and then something obviously a more desperate incident would pop up and they'd say, "No, we can't do it now. Don't eat, we'll do it in the morning" ... So I became quite desperate and I lost quite a bit of weight. (35-year-old man, Medicare, multiple injuries)

Communication: Most participants reported issues with communication and receipt of information. In particu- lar, ward rounds were highlighted because of their timing, lack of engagement of the patient and the impersonal nature of the process. The issues with communication were:

1. A lack of information about prognosis or incorrect prognosis;

2. Inability to absorb information because of the effects of the injury and treatment (eg, medication);

3. Insufficient explanation of the risks of treatment options, or providing treatment options without sufficient information to make an informed decision;

4. Conflicting information provided by clinicians; and

5. Limited engagement of the patient in decision making.

I would have probably liked a little more explanation at the start of exactly what the injury entailed. I understand the job of the doctors is to keep patients positive I guess, but I was told many times that the bone will be healed in 6 weeks' time. And I was told that about four or five times. (26-year-old man, $\mathrm{TAC}$, multiple fractures)

... That morning there was also a massive ward round with probably six medical students and a whole lot of doctors. And so I didn't really feel so able to ask the questions I wanted to ask. I didn't even quite realise who to talk to, like who's the boss out of the 12 people standing around my bed? (34-year-old woman, TAC, extremity fracture)

\section{Hospital discharge and post-discharge care}

Discharge from hospital care was a stressful time for a number of participants, and many felt ill prepared for it either emotionally, physically, or as a result of insufficient information about their limitations and post-discharge care.

When they send you home, I think just your whole world's been turned upside down and any instruction or anything like that that you're given, you just don't remember at all. (37-year-old woman, TAC, spinal fractures)

Forty-five (38\%) participants were discharged to inpatient rehabilitation facilities. Participants reported opting not to go to rehabilitation because of the distance family members had to travel to visit. Others expressed their 
need for rehabilitation but this option was not offered. Inpatient rehabilitation experiences varied depending on the specific facility and perceived need.

... the programs that they had ... were very good for people like me. There was everything there and you're on a daily assessment, and I think that was just getting back into a routine of some description was very good for me. (62-year-old woman, Medicare, head injury)

... And then they said, "You have to go to rehabilitation", which I wasn't very impressed with ... I spent 6 weeks there and I hardly had more than 4 hours of therapy a week; it was just a waste of the time and a waste of bed ... (67-year-old man, private, extremity fractures)

Lack of coordinated care: A consistent theme emerging from the post-discharge care of patients was the sense of a lack of coordination of care, and absence of a consistent point of contact for patients about their ongoing management. For most, their primary point of post-discharge contact was outpatient clinics at the MTS hospitals. These were widely criticised because of substantial delays in receiving an appointment, prolonged waiting times, limited time with clinicians, lack of continuity of care, and limited exposure to senior clinicians. A number of participants opted for private care to avoid the outpatient clinics.

The outpatient system is an absolute disgrace. I live ... $200 \mathrm{~km}$ from the (hospital). I drive down there, sit in traffic, pay exorbitant amounts for parking, sit in the waiting room for over 3 hours and then spend $10 \mathrm{~min}$ utes with a different doctor to the one that was there last time ... (37-yearold man, TAC, extremity fracture)

... and I've spent 5 hours here to speak to a very junior doctor and get dodgy advice. ... I'm going to go private. (34-year-old woman, TAC, extremity fracture)

Many participants expressed the need for a restructure of outpatient delivery. Others provided practical recommendations for improving patient experiences:

1. Informing patients about delays;

2. Investigating systems for early notification of patients about delays (eg, SMS messaging);
3. Improved use of remote and telemedicine approaches, particularly for patients remote from central services;

4. Consultations of suitable duration to address patient questions; and

5. Improved continuity of care through consistency of clinician contact for follow-up (eg, see the same surgeon each time).

\section{Discussion}

This study is the first to provide a detailed description of patient experiences with the Victorian MTS and trauma care providers. It highlights perceived issues and limitations in the patient care pathway in the VSTS, especially relating to communication, information provision and post-discharge care.

Poor communication with service providers was a common theme, and is a longstanding health care issue. ${ }^{16,17}$ There were difficulties in identifying the most appropriate contact point for information in hospital, not helped by the ward round system. Another study found that while clinical staff perceived the round to be intimidating and embarrassing for patients, this was not the perception of patients. ${ }^{18}$ In our study, participants found it intimidating and unsatisfactory.

Participants reported a stark contrast between their experience of recovery and clinician prognosis, leading to frustration and dissatisfaction with their outcomes. They also felt they received contradictory information about treatment options. Many felt insufficiently informed to make decisions about their treatment and rehabilitation choices, leading to a lack of trust in their clinical management. The need for improved information and prognostication has previously been highlighted. ${ }^{19,20}$

Provision of a seamless transition from acute hospital care to community and rehabilitation services is challenging. ${ }^{19}$ The blueprint of the VSTS, the Review of Trauma and Emergency Services report, identified the role of the MTS as coordinating the total care of the patient right through to rehabilitation. ${ }^{7}$ In our study, participants perceived their hospital care as high quality. However, there was a strong
2 Characteristics of 120 trauma patients treated at major trauma services in Victoria

\begin{tabular}{|c|c|}
\hline Mean age, years (SD) & $48.6(17.6)$ \\
\hline 18-44 years & $48(40.0 \%)$ \\
\hline 45-64 years & 48 (40.0\%) \\
\hline$\geqslant 65$ years & $24(20.0 \%)$ \\
\hline Male & $63(52.5 \%)$ \\
\hline Female & $57(47.5 \%)$ \\
\hline \multicolumn{2}{|l|}{ Pre-injury employment } \\
\hline Working or studying & $85(70.8 \%)$ \\
\hline Not working or studying & $35(29.2 \%)$ \\
\hline \multicolumn{2}{|l|}{ Pre-injury disability } \\
\hline None & $98(81.7 \%)$ \\
\hline Mild & $13(10.8 \%)$ \\
\hline Moderate to severe & $9(7.5 \%)$ \\
\hline \multicolumn{2}{|l|}{ Hospital of definitive care } \\
\hline The Alfred & $60(50.0 \%)$ \\
\hline Royal Melbourne Hospital & $60(50.0 \%)$ \\
\hline \multicolumn{2}{|l|}{ Registry } \\
\hline Victorian State Trauma Registry & $62(51.7 \%)$ \\
\hline Victorian Orthopaedic Trauma Outcomes Registry & $58(48.3 \%)$ \\
\hline Median time since injury in months (range) & $14.2(12.8-19.7)$ \\
\hline \multicolumn{2}{|l|}{ Fund source } \\
\hline Transport Accident Commission & $62(51.7 \%)$ \\
\hline Medicare & $40(33.3 \%)$ \\
\hline Private & $16(13.3 \%)$ \\
\hline WorkCover & $2(1.7 \%)$ \\
\hline \multicolumn{2}{|l|}{ Mechanism of injury } \\
\hline Motor vehicle & 24 (20.0\%) \\
\hline Motorcycle & $21(17.5 \%)$ \\
\hline Low fall & 19 (15.8\%) \\
\hline Pedal cyclist & $16(13.3 \%)$ \\
\hline High fall & $13(10.8 \%)$ \\
\hline Pedestrian & $6(5.0 \%)$ \\
\hline Struck by/collision with object or person & $5(4.2 \%)$ \\
\hline Horse-related & $4(3.3 \%)$ \\
\hline Other & $12(10.0 \%)$ \\
\hline $\begin{array}{l}\text { Median hospital length of stay in days } \\
\text { (interquartile range) }\end{array}$ & $6.2(2.3-11.6)$ \\
\hline \multicolumn{2}{|l|}{ Discharge destination } \\
\hline Home & 75 (62.5\%) \\
\hline Inpatient rehabilitation & 45 (37.5\%) \\
\hline
\end{tabular}

perception of a lack of coordination between MTS acute care and postdischarge services, exacerbated by the rapidity of hospital discharge.

For most, the primary contact point after discharge was MTS outpatient clinics, which drew almost universal criticism from participants for considerable waits and unmet expectations and needs, raising the question about the most appropriate post-discharge services for seriously injured patients. The patients' need for a central point of contact for coordination of ongoing care was raised but who this should be was not clear. Participants felt that their general practitioner could fulfil 
3 Twelve-month outcomes for 120 trauma patients treated at major trauma services in Victoria

Extended Glasgow Outcome Scale score

Upper good recovery (return to pre-injury capacity for work or study, social and leisure activities)

Lower good recovery (return to pre-injury capacity for work or study, but less participation in social and leisure activities)

Upper moderate disability (return to work or study at reduced capacity, but with restricted participation in social and

leisure activities)

Lower moderate disability (able to shop and get around the community but unable to work or study, and rarely participates

in social or leisure activities)

Severe disability (needs assistance at home and unable shop or travel locally without assistance)

Return to work (proportion of those who were working before injury)

12-item Short Form Health Survey scores

Mean Physical Component Summary score (SD)

Mean Mental Component Summary score (SD)

this role, particularly those for whom routine follow-ups raised logistical issues. Options such as telemedicine may assist patients in these circumstances, but the shortage of GPs, especially in rural areas, could be a limiting factor.

To our knowledge, this is the largest qualitative study of trauma patients' perceptions of care worldwide. Although undertaken in the VSTS, many of the participants' issues apply to other health care contexts. The study was limited by some undersampling in specific groups and by its focus on adult patients managed at the MTS hospitals (where most major trauma patients are managed), which may not reflect the wider, less severely injured patients in the VSTS population. Nevertheless, these narratives provided an unparalleled insight into the issues faced by injured participants, contributing to our understanding of how to improve services, especially after discharge.

Acknowledgements: This project was funded by the TAC through a development grant from the Institute for Safety Compensation and Recovery Research (ISCRR). The VSTR is a funded initiative of the Department of Health Victoria and TAC. The VOTOR is funded by the TAC via the ISCRR. We would like to thank the investigators, Steering Committees and the participating hospitals of the VSTR and VOTOR. We would also like to thank Helen Donaldson and Libby Richards for their assistance with the interviews, and Dina Watterson for her involvement in the project.

Competing interests: No relevant disclosures.

Received 26 Jul 2012, accepted 5 Nov 2012.

1 Meredith JW. An important next step in the evolution of our understanding of the value and importance of trauma systems. Ann Surg 2012; 255: 1016.

2 Cameron PA, Gabbe BJ, Cooper DJ, et al. A statewide system of trauma care in Victoria: effect on patient survival. Med J Aust 2008; 189: 546-550.

3 MacKenzie EJ, Rivara FP, Jurkovich GJ, et al. A national evaluation of the effect of traumacenter care on mortality. New Engl J Med 2006; 354: 366-378.

4 Gabbe BJ, Simpson PM, Sutherland AM, et al. Improved functional outcomes for major trauma patients in a regionalized, inclusive trauma system. Ann Surg 2012; 255: 1009-1015.

5 MacKenzie EJ, Rivara FP, Jurkovich GJ, et al. The impact of trauma-center care on functional outcomes following major lower-limb trauma. J Bone Joint Surg Am 2008; 90: 101-109.

6 MacKenzie EJ, Rivara FP, Jurkovich GJ, et al. The national study on costs and outcomes of trauma. J Trauma 2007; 63 (6 Suppl): S54-S67.

7 Ministerial Taskforce on Trauma and Emergency Services and the Department of Human Services Working Party on Emergency and Trauma Services. Review of trauma and emergency services 1999. Melbourne: Department of Human Services, 1999. http://www.health.vic.gov.au/ trauma/review99/index.htm (accessed Jul 2012).

8 Cameron PA, Finch CF, GabbeBJ, et al. Developing Australia's first statewide trauma registry: what are the lessons? ANZ J Surg 2004; 74: 424-428.

9 Gabbe BJ, Cameron PA, Williamson OD, et al. The relationship between compensable status and long-term patient outcomes following orthopaedic trauma. Med J Aust 2007; 187: 14-17.

10 Gooberman-Hill R, Fox R. What can qualitative approaches bring to trauma outcome research? Injury 2011; 42: 321-323.

11 Edwards ER, Graves SE, McNeil JJ, et al; Victorian Orthopaedic Trauma Outcomes Registry Project Group. Orthopaedic trauma: establishment of an outcomes registry to evaluate and monitor treatment effectiveness. Injury 2006; 37: 95-96.

12 Gabbe BJ, Sutherland AM, Hart MJ, Cameron PA. Population-based capture of long-term functional and quality of life outcomes after major trauma: the experiences of the Victorian State Trauma Registry. J Trauma 2010; 69: 532-536.

13 Barbour RS. Checklists for improving rigour in qualitative research: a case of the tail wagging the dog? BMJ 2001; 322: 1115-1117.

14 Pope C, Mays N. Qualitative research in health care. 3rd ed. Oxford: Blackwell Publishing, 2006.

15 Green J, Thorogood N. Qualitative methods for health research. 2nd ed. London: Sage, 2009.

16 Simpson M, Buckman R, Stewart M, et al. Doctorpatient communication: the Toronto consensus statement. BMJ 1991; 303: 1385-1387.

17 Politi MC, Street RL Jr. The importance of communication in collaborative decision making: facilitating shared mind and the management of uncertainty. J Eval Clin Pract 2011; 17: 579-584.

18 Mahar P, Lake H, Waxman BP. Patient perceptions of the surgical ward round. $A N Z$ Surg 2009; 79: 584-585.

19 Ameratunga S, Abel S, Tin Tin S, et al. Children admitted to hospital following unintentional injury: perspectives of health service providers in Aotearoa/New Zealand. BMC Health Serv Res 2010; 10: 333.

20 Harms L. After the accident: survivors' perceptions of recovery following road trauma. Aust Social Work 2004; 57: 161-174. doi: 10.1111/ j.1447-0748.2004.00129.x. 